\title{
PROPOSIÇÃO DE UM ÍNDICE DE PRODUÇÃO PARA BIBLIOTECAS COM USO DO MÉTODO MACBETH
}

\author{
Eliane Gonçalves Gomes ${ }^{1}$ \\ Maria de Cléofas Faggion Alencar ${ }^{2}$
}

\section{Resumo}

Este artigo apresenta o uso de um método multicritério para propor um índice de produção ponderado para bibliotecas. O índice é composto dos diversos tipos de serviços prestados pelas bibliotecas e para agregá-los foram utilizados pesos gerados a partir dos julgamentos de valor de um bibliotecário sobre a importância relativa entre os serviços. Para transformar os julgamentos qualitativos em quantitativos foi usado o método multicritério MACBETH. O índice proposto pode servir como medida de comparação entre diversas bibliotecas, em especial quando estão sujeitas a uma administração central.

Palavras-chave: Multicritério; MACBETH; Serviços de biblioteca; Indicadores.

\begin{abstract}
In this paper we present a weighted production indicator to evaluate libraries. This indicator is composed by the various kinds of services offered in the libraries. In order to aggregate these services, we used the weights obtained from a librarian value judgements about their relative importance. To transform qualitative judgements into quantitative ones, we used the MACBTEH multicriteria approach. The proposed indicator can be used as a comparative evaluation score, particularly when libraries are subjected to a central administration.
\end{abstract}

Key words: Muticriteria; MACBETH; Library services; Indicators.

\section{INTRODUÇÃO}

Desde o século XIX, o objetivo principal das bibliotecas é proporcionar aos seus usuários um serviço: o do acesso à informação. Atualmente, a diferença está nos canais adicionais e distintos pelos quais oferece-se o serviço de biblioteca e o desafio está em determinar como, quando e se, esses meios deveriam ser usados. Por isso, as bibliotecas têm tido sérios problemas com a aquisição, o armazenamento, o manuseio de documentos e seus registros, já que quando se trata de mudanças tecnológicas, os efeitos e conseqüências são ainda mais significativas para os procedimentos de aquisição, armazenamento, entrega e localização de informação.

Buckland (1992) sugere bases gerais de planejamento e/ou uma estrutura geral para repensar os serviços de biblioteca do futuro e, dessa forma,

\footnotetext{
1 Embrapa Sede - Secretaria de Gestão e Estratégia; Parque Estação Biológica, W3 Norte final, Asa Norte, 70770-901, Brasília, DF; eliane.gomes@embrapa.br

2 Embrapa Monitoramento por Satélite; Av. Dr. Júlio Soares de Arruda, 803, Parque São Quirino, 13088300, Campinas, SP; cleo@cnpm.embrapa.br
} 
preparar-se para retratar os primeiros princípios: “O serviço de biblioteca é uma atividade constante, orientada para o serviço, com uma ênfase profundamente enraizada e refletida na literatura profissional, em assuntos práticos e técnicos, nos meios mais do que nos fins e no nível tático mais do que no estratégico".

Uma análise comparada entre artigos publicados em periódicos das áreas de Biblioteconomia e Ciência da Informação entre 1990 e 1999, mostra que $44,4 \%$ dos artigos publicados no Brasil contra $86,1 \%$ dos artigos internacionais são de temas relacionados aos seguintes serviços de bibliotecas: organização e gerência de atividades, serviços técnicos, entradas, tratamento, armazenamento, recuperação e disseminação de informação (MUELLER; PECEGUEIRO, 2001). Uma das razões para a concentração de artigos publicados sobre o tema "serviços de bibliotecas" pode ser atribuída à preocupação e à tentativa de acompanhamento pela comunidade acadêmica e de profissionais dessas áreas ao crescimento do setor de serviços na economia mundial. A busca de melhoria da qualidade está particularmente localizada na gestão de serviços.

Nesse contexto, como avaliar os serviços de bibliotecas tornou-se uma questão importante, em especial porque eles têm importância diferenciada. Se esses serviços são prestados por bibliotecas de um sistema, como é o caso das bibliotecas do Sistema de Bibliotecas da Empresa Brasileira de Pesquisa Agropecuária (Embrapa), que conta hoje com 38 Bibliotecas, situadas em suas unidades centrais e descentralizadas, essa avaliação torna-se ainda mais relevante, em especial para a gestão feita pela administração central.

Este artigo tem como objetivo fazer uma primeira proposta de um índice de produção de serviços para as bibliotecas. Como cada serviço tem importância diferenciada, este índice é um índice ponderado, em que cada peso tenta expressar a importância relativa de cada serviço na composição do "serviço ENGEVISTA, v. 7, n. 1, p. 21-31, abril 2005 global”. O índice é composto pelos diversos tipos de serviços prestados pelas bibliotecas. Para agregá-los foram utilizados os julgamentos de valor da bibliotecária de uma das unidades descentralizadas da Embrapa. Para transformar os julgamentos de valor qualitativos em quantitativos foi usado o método multicritério MACBETH (BANA E COSTA; VANSNICK, 1995, 1997). O índice proposto pode servir como medida de comparação entre diversas bibliotecas, em particular quando sujeitas a uma administração central.

\section{MODELAGEM PROPOSTA}

\subsection{Apoio Multicritério à Decisão}

O Apoio Multicritério à Decisão (AMD) consiste em um conjunto de método e técnicas para auxiliar ou apoiar pessoas e organizações a tomarem decisões, quando da presença de uma multiplicidade de critérios (GOMES et al., 2002). A aplicação de qualquer método de análise multicritério pressupõe a especificação do objetivo que o decisor pretende alcançar, quando deseja comparar entre si alternativas de decisão recorrendo ao uso de múltiplos critérios.

No AMD buscam-se construir modelos que legitimem os juízos de valor subjetivos, ou seja, pressupõe aceitar que a subjetividade estará presente em todo o processo de decisão. Dessa forma, a estrutura de valores dos decisores é associada aos critérios existentes, e que serão usados na avaliação das alternativas (YU, 1985).

A análise de decisão com múltiplos critérios é um processo iterativo, mas pode ser apresentado como a seqüência das seguintes etapas (GOMES et al., 2004): 1) Identificar os decisores e seus objetivos; 2) Definir as alternativas; 3) Definir os critérios relevantes para o problema de decisão; 4) Avaliar alternativas em relação aos critérios; 5) Determinar importância relativa dos critérios; 6) Realizar a avaliação global de cada alternativa; 7) Análise de sensibilidade; Recomendação; 9) Implementação. 
As etapas 1, 2 e 3 são conhecidas como Fase de Estruturação do problemas, a qual segundo Bana e Costa et al. (2000), representa cerca de $80 \%$ do total do problema. Esta fase trata da formulação do problema e busca identificar, caracterizar e organizar os fatores considerados relevantes no processo de apoio à decisão. É uma fase interativa e dinâmica, pois fornece uma linguagem comum aos decisores, o que possibilita a aprendizagem e o debate.

As etapas 4, 5, 6 e 7 compõem a Fase de Avaliação, que tem como objetivo a aplicação de métodos de análise multicritério para apoiar a modelagem das preferências e a sua agregação. A terceira fase, composta das etapas 8 e 9, é a Fase de Recomendação dos cursos de ação a serem seguidos.

Em relação à etapa 3 , cabe ressaltar que em um problema de decisão complexo, os critérios podem ser estruturados na forma de hierarquia ou árvore, na qual o nível do critério mais elevado é decomposto em níveis mais detalhados (GOMES et al., 2004).

Deve-se ainda atentar que uma família de critérios, ou seja, o conjunto de critérios utilizados em uma determinada situação de decisão, deve satisfazer a três condições ("axiomas de Roy") para que seja uma família coerente de critérios (ROY, BOUYSSOU, 1993; SOARES DE MELLO et al., 2003): Exaustividade (impõe a necessidade de descrever o problema levando em conta todos os aspectos relevante); Coesão (obriga à correta análise de quais são os critérios de maximização e quais os de minimização); Não Redundância (obriga a excluir critérios que estejam avaliando características já avaliadas por outro critério).

Em AMD os juízos de valor dos decisores são expressos por meio de suas Estruturas de Preferência entre pares de alternativas ou critérios. As quatro relações fundamentais são indiferença, preferência estrita, preferência fraca e incomparabilidade (ROY, BOUYSSOU, 1993; DIAS et al., 1996).

A forma de explicitar as estruturas de preferência do decisor varia de acordo ENGEVISTA, v. 7, n. 1, p. 21-31, abril 2005 com o método de análise multicritério escolhido. Para a construção do índice de produção das bibliotecas foi escolhido um método que pode ser considerado como da escola americana de multicritério (GOMES et al., 2004; POMEROL, BARBA-ROMERO, 2000; GOMES, 1999), já que o objetivo é construir um índice único que agregue, por meio de pesos (também chamados de constantes de escala ou taxas de substituição), os diversos serviços prestados pela biblioteca da Embrapa Monitoramento por Satélite. O método escolhido é o MACBETH (Measuring Attractiveness by a Categorical Based Evaluation Technique).

\subsection{MACBETH}

$\begin{array}{ccr}\text { O método MACBETH } \\ \text { (Measuring } & \text { Attractiveness by a } \\ \text { Categorical } & \text { Based Evaluation } \\ \text { Technique), desenvolvido por Bana e }\end{array}$
Costa, Vansnick (1995, 1997) e apresentado em Bana e Costa et al. (2003, 2004) e Bana e Costa, Chagas (2004), permite agregar os diversos critérios de avaliação em um critério único de síntese por meio da atribuição de pesos aos vários critérios, respeitando as opiniões dos decisores.

Com a comparação par a par da atratividade das alternativas são atribuídos os pesos aos critérios: dadas duas alternativas, o decisor deve dizer qual a mais atrativa (deve receber a maior nota) e qual o grau desta atratividade em uma escala semântica que tem correspondência com uma escala ordinal ( $0 \equiv$ indiferente, $1 \equiv$ diferença de atratividade muito fraca, 2 三 diferença de atratividade fraca, $3 \equiv$ diferença de atratividade moderada, $4 \equiv$ diferença de atratividade forte, $5 \equiv$ diferença de atratividade muito forte e $6 \equiv$ diferença de atratividade extrema).

O software que implementa computacionalmente o método faz a análise de coerência dos julgamentos e sugere, em caso de incoerência, como resolvê-la. Por programação linear é sugerida uma escala de notas, e os intervalos em que elas podem variar sem 
tornar o problema inconsistente (Problema de Programação Linear inviável). É ainda facultado ao decisor ajustar graficamente o valor das notas atribuídas, dentro dos intervalos permitidos (análise de sensibilidade). Segundo Bana e Costa, Vansnick (1997) somente após este ajuste, com a introdução dos conhecimentos dos especialistas, é que fica caracterizada a construção da escala cardinal (quantitativa) de valores.

Matematicamente, a metodologia MACBETH é constituída por quatro Problemas de Programação Linear (PPLs) seqüenciais:

- PPL 1 - Problema Mc1: realiza a análise de consistência cardinal;

- PPL 2 - Problema Mc2: responsável pela construção da escala de valor cardinal;

- PPLs 3 e 4 - Problemas Mc3 e Mc4: revelam fontes de inconsistência.

Para operacionalizar o método, Bana e Costa, Vansnick (1995) propõem a construção de matrizes de juízos de valor para facilitar a expressão dos julgamentos absolutos de diferença de atratividade entre os pares de ações. Cada elemento $x_{i j}$ da matriz toma o valor $k$ ( $k=$ $1,2,3,4,5,6)$ se o decisor julgar que a diferença de atratividade do par $\left(a_{i}, a_{j}\right)$ pertence à categoria $C_{k}$. Estes números não têm significado matemático; servem apenas como indicadores semânticos de qual categoria de diferença de atratividade foi atribuída ao par respectivo.

Com este conjunto de julgamentos, a metodologia MACBETH é executada, primeiramente para a verificação de eventuais inconsistências e, posteriormente, para a determinação de uma escala de valor cardinal que represente os julgamentos de valor do decisor. A escala obtida é normalizada, fornecendo os valores dos pesos para as alternativas em avaliação, o que possibilita o uso de um modelo de agregação, em geral, aditivo.

\subsection{Modelagem}

Como colocado anteriormente, a primeira fase de uma modelagem ENGEVISTA, v. 7, n. 1, p. 21-31, abril 2005 multicritério é a fase de estruturação, composta de três etapas. $\mathrm{Na} 1^{\mathrm{a}}$ etapa foram identificados os decisores e seus objetivos. O agente que atuou como decisor foi a bibliotecária da Embrapa Monitoramento por Satélite, isto é, foi quem emitiu os juízos de valor acerca das diferenças de atratividade entre as ações avaliadas. O objetivo do decisor é gerar um índice de produção único que agregue todos os serviços prestados pela biblioteca.

Nessa fase foram ainda identificadas as ações a serem avaliadas. Essa família de ações deve ser uma família coerente, ou seja, deve ser coesa, não redundante e exaustiva. A árvore de ações obtida é apresenta na Figura 1. Os ramos da árvore foram cortados quando não havia diferença de atratividade entre as subações da ação em questão.

Na segunda fase, ou seja, na fase de avaliação, foi aplicado o método multicritério MACBETH que apoiou na modelagem das preferências e na agregação das ações (serviços das bibliotecas). Essa etapa foi realizada no sentido ramos-tronco da árvore, ou seja, foi feita uma avaliação para os subprocessos e posteriormente para os processos.

Cabe destacar que a árvore da Figura 1 foi construída com base na experiência da bibliotecária da Embrapa Monitoramento por Satélite (decisor) e nos serviços prestados por essa biblioteca. Outras bibliotecas podem apresentar árvores de serviços diferentes desta. Ressalta-se, ainda, que apesar de a estrutura hierárquica da Figura 1 sugerir, a princípio, o uso do método AHP Analytic Hierarchy Process (SAATY, 1980), este foi preterido em relação ao método MACBETH pelo fato de este não permitir inconsistência nos julgamentos do decisor (estabelece um processo interativo de revisão dos julgamentos e sugere, se necessário, quais devem ser revistos). Já o método AHP arbitra um valor de $10 \%$ para a inconsistência máxima nos julgamentos. Bana e Costa, Vansnick (2001) apresentam uma discussão sobre alguns problemas relacionados ao uso do método AHP. 


\section{RESULTADOS E DISCUSSÃO}

\subsection{Avaliação dos subprocessos}

\subsubsection{Subprocessos do processo} "Manutenção do acervo"

O processo "Manutenção do acervo" pode ser dividido em quatro subprocessos de importância diferenciada.

Primeiramente, o método MACBETH pede uma hierarquização por ordem crescente de atratividade desses procedimentos. $\mathrm{O}$ decisor propôs a seguinte ordenação: Seleção; Aquisição; Desbastamento; Restauração e Encadernação.

A segunda etapa é emitir os julgamentos de valor sobre as diferenças de atratividade entre cada par de subprocessos do processo "manutenção do acervo". É construída uma matriz que incorpora os julgamentos. A Figura 2 mostra essa matriz e os julgamentos. Conforme já mencionado, é incluído um processo fictício que representa a pior situação dentre todas; nesse caso, por exemplo, seria não executar o processo de manutenção.

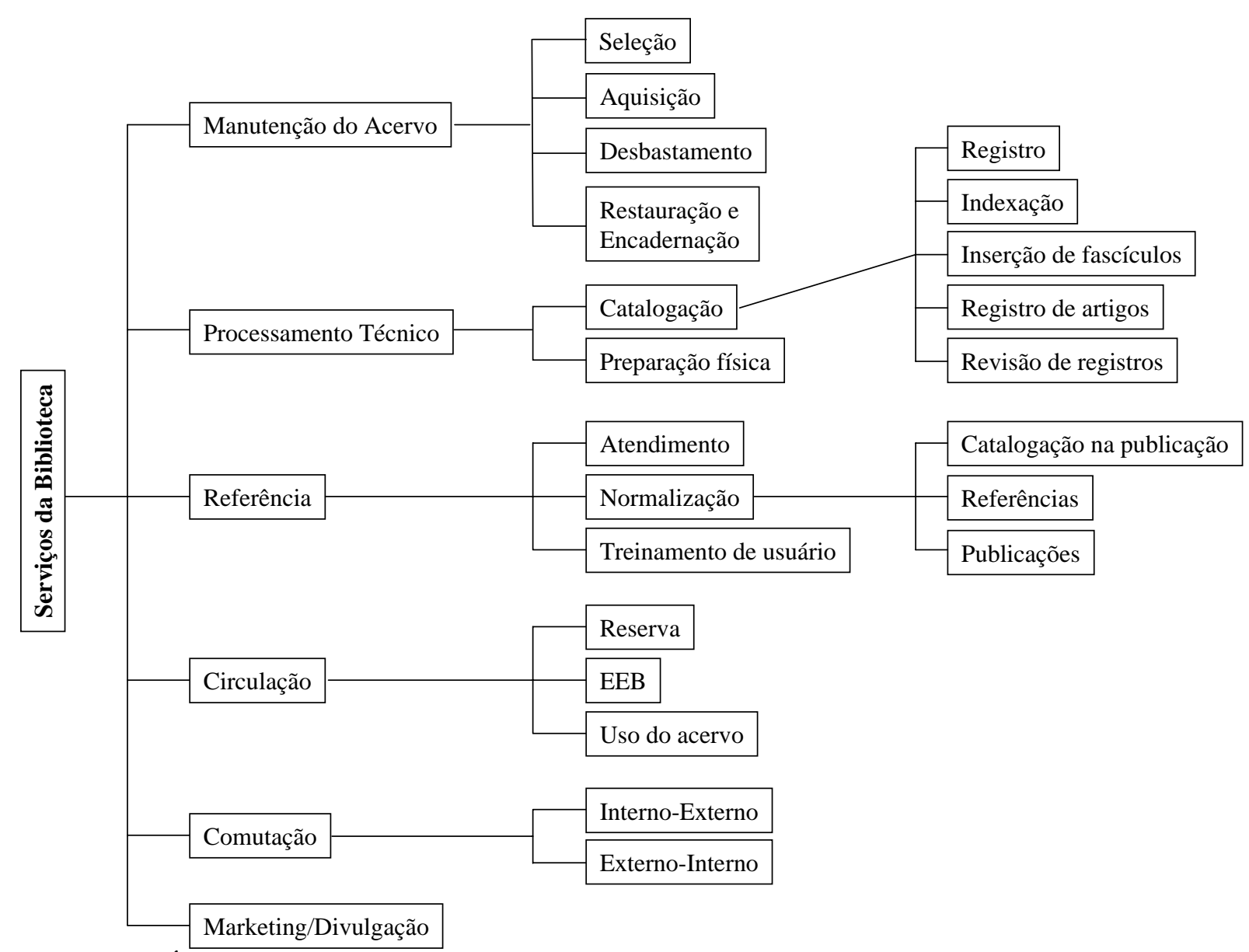

Figura 1. Árvore de serviços/processos da biblioteca da Embrapa Monitoramento por Satélite. 


\begin{tabular}{|l|c|c|c|c|c|}
\cline { 2 - 6 } \multicolumn{1}{c|}{} & Seleção & Atualização & Desbastamento & $\begin{array}{c}\text { Restauração e } \\
\text { Encadernação }\end{array}$ & $\begin{array}{c}\text { Não manter o } \\
\text { acervo }\end{array}$ \\
\hline Seleção & --- & Indiferente & Forte & Muito forte & Extrema \\
\hline Atualização & --- & --- & Forte & Muito forte & Extrema \\
\hline Desbastamento & --- & --- & --- & Moderada & Extrema \\
\hline $\begin{array}{l}\text { Restauração e } \\
\text { Encadernação }\end{array}$ & --- & --- & --- & --- & Extrema \\
\hline $\begin{array}{l}\text { Não manter o } \\
\text { acervo }\end{array}$ & --- & --- & --- & --- & --- \\
\hline
\end{tabular}

Figura 2: Matriz de julgamentos de valor para os subprocessos do processo "Manutenção do Acervo".

Cada elemento da matriz é preenchido com o julgamento de valor. Por exemplo, o preenchimento do elemento da matriz que avalia a diferença de atratividade entre os subprocessos de Seleção e Atualização é preenchido com a reposta a seguinte pergunta: Dado que para o processo de Manutenção do acervo, passar a atividade de Seleção do nível neutro para o nível bom é mais atrativo que passar a atividade de Atualização do nível neutro para o nível bom, qual é essa diferença de atividade (indiferente, muito fraca, fraca, moderada, forte, muito forte, extrema)?

A pergunta é feita de modo a completar todos os elementos da matriz. Destaca-se que os julgamentos acerca dos níveis neutro e bom só foram possíveis após a definição, pelo decisor, ao que correspondem. Como os processos a serem avaliados já encontram-se em ordem decrescente de atratividade, só é necessário preencher a matriz triangular superior (a outra parte da matriz representaria os julgamentos inversos).
A partir da construção dessa matriz, o método MACBETH é conduzido (resolução dos 4 PPLs seqüenciais) e são gerados os pesos para cada um dos processos.

Com os julgamentos de valor expressos na Figura 2, os pesos gerados pelo MACBETH para cada processo são: Seleção = 30,61\% (SEL); Aquisição = 30,61\% (AQ); Desbastamento = 22,45\% (DESB); Restauração e Encadernação = 16,33\% (ReE). É ainda facultado ao decisor executar uma análise se sensibilidade que permite alterar os valores dos pesos, dentro dos limites estabelecidos pelos julgamentos de valor e análises de consistência (observe-se que ao alterar um dos limites para um dos processo, todos os valores de pesos para os demais processos são alterados, de modo a manter soma igual a $100 \%$ ). A Tabela 1 apresenta os limites superior e inferior para esse caso.

Tabela 1: Limites superior, inferior e valor sugerido para os pesos do processo "Manutenção do Acervo".

\begin{tabular}{|l|c|c|c|}
\cline { 2 - 4 } \multicolumn{1}{c|}{} & Limite inferior & Peso sugerido & Limite superior \\
\hline Seleção & 29,81 & 30,61 & 31,35 \\
\hline Aquisição & 29,81 & 30,61 & 31,35 \\
\hline Desbastamento & 17,42 & 22,45 & 23,21 \\
\hline Restauração e Encadernação & 15,49 & 16,33 & 21,13 \\
\hline Não manter o acervo & 0,00 & 0,00 & 0,00 \\
\hline
\end{tabular}

Matematicamente, 0 processo "Manutenção do acervo" pode ser visto como a agregação ponderada aditiva dos quatro subprocessos, conforme expressão (1). 
3.1.2. Subprocessos do processo "Processamento Técnico"

O processo Processamento Técnico foi dividido nos processos de Catalogação (CAT) e Preparação Física (PF). Já o processo de Catalogação foi ainda dividido em cinco subprocessos, já em ordem decrescente de atratividade: Registro (REG); Indexação (IND); Registro de Artigo de Periódicos (RAP); Revisão de Registros (RR); Inserção de Fascículo de Periódicos (IFP).

Foi efetuado o mesmo procedimento explicitado anteriormente, de emissão de juízos de valor pelo decisor sobre as diferenças de atratividade entre pares de processos. Nesse caso, o procedimento foi feito para dois conjuntos: o referente ao processo de Catalogação e aquele referente ao processamento técnico.

As Tabelas 2 e 3 apresentam os valores dos pesos. As expressões (2) e (3) mostram matematicamente as relações derivadas.

Tabela 2: Pesos para as atividades do subprocesso "Catalogação".

\begin{tabular}{|l|c|c|c|}
\cline { 2 - 4 } \multicolumn{1}{c|}{} & Limite inferior & Peso sugerido & Limite superior \\
\hline Registro & 22,60 & 23,71 & 24,22 \\
\hline Indexação & 22,60 & 23,71 & 24,22 \\
\hline Registro de artigo & 21,26 & 21,65 & 23,21 \\
\hline Revisão de registros & 18,15 & 18,56 & 19,37 \\
\hline Inserção de fascículos & 11,94 & 12,37 & 13,25 \\
\hline
\end{tabular}

Tabela 3: Pesos para os subprocessos do processo "Processamento técnico".

\begin{tabular}{|l|c|c|c|}
\cline { 2 - 4 } \multicolumn{1}{c|}{} & Limite inferior & Peso sugerido & Limite superior \\
\hline Catalogação & 50,04 & 66,67 & 99,97 \\
\hline Preparação física & 0,06 & 33,33 & 49,97 \\
\hline
\end{tabular}

$\mathrm{CAT}=23,71 * \mathrm{REG}+23,71 * \mathrm{IND}+21,65 * \mathrm{RAP}+28,56 * \mathrm{RR}+12,37 * \mathrm{IFP}$

Processamento técnico $=66,67 * \mathrm{CAT}+33,33 * \mathrm{PF}$

\subsubsection{Subprocessos do processo} "Referência"

O processo Referência foi dividido nos processos de Atendimento (ATD), Normalização (NORM) e Treinamento de usuários (TU), já em ordem decrescente de atratividade. Já o processo de normalização foi ainda subdividido em três processos: Catalogação na publicação - CIP (CIP);
Referências (REFs); Publicações (PUBs) (em ordem decrescente de atratividade).

Os mesmos procedimentos foram realizados para os dois conjuntos (referente ao subprocesso de Normalização e o referente ao processo de Referência).

As Tabelas 4 e 5 apresentam os valores dos pesos. As expressões (4) e (5) mostram matematicamente as relações derivadas.

Tabela 4: Pesos para as atividades do subprocesso "Normalização".

\begin{tabular}{|l|c|c|c|}
\cline { 2 - 4 } \multicolumn{1}{c|}{} & Limite inferior & Peso sugerido & Limite superior \\
\hline Catalogação na publicação & 37,16 & 42,11 & 43,57 \\
\hline Referências & 33,36 & 34,21 & 39,01 \\
\hline Publicações & 21,65 & 23,68 & 25,62 \\
\hline ENGEVISTA, v. 7, n. 1, p. 21-31, abril 2005
\end{tabular}


Tabela 5: Pesos para os subprocessos do processo "Referência”.

\begin{tabular}{|l|c|c|c|}
\cline { 2 - 4 } \multicolumn{1}{c|}{} & Limite inferior & Peso sugerido & Limite superior \\
\hline Atendimento & 41,69 & 48,15 & 19,98 \\
\hline Normalização & 30,80 & 33,33 & 41,91 \\
\hline Treinamento usuário & 15,42 & 18,52 & 22,78 \\
\hline
\end{tabular}

$\mathrm{NORM}=42,11 * \mathrm{CIP}+34,21 *$ REFs $+23,68 *$ PUBs

Referência $=48,15 *$ ATD $+33,33 *$ NORM +18,52*TU

3.1.4. Subprocessos do processo "Circulação"

O processo Circulação foi dividido nos processos de Uso do Acervo (UA), Empréstimo entre Bibliotecas
(EEB) e Reserva (RES), em ordem decrescente de atratividade.

A Tabela 6 apresenta os valores dos pesos e a expressão (6) a formalização matemática desses relacionamentos.

Tabela 6: Pesos para as atividades do processo "Circulação".

\begin{tabular}{|l|l|l|l|}
\cline { 2 - 4 } \multicolumn{1}{c|}{} & Limite inferior & Peso sugerido & Limite superior \\
\hline Uso do Acervo & 50,03 & 55,56 & 99,92 \\
\hline EEB & 25,04 & 33,33 & 39,97 \\
\hline Reserva & 0,05 & 11,11 & 15,75 \\
\hline
\end{tabular}

Circulação $=50,03 *$ UA + 33,33* EEB + 11,11* RES

3.1.5. Subprocessos do processo "Comutação"

O processo Comutação foi dividido nos processos de comutação
Interno-Externo (IE) e os processos Externo-Interno (EI).

A Tabela 7 e a expressão 7 resumem os resultados da avaliação deste processo.

Tabela 7: Pesos para as atividades do processo "Comutação".

\begin{tabular}{|l|c|c|c|}
\cline { 2 - 4 } \multicolumn{1}{c|}{} & Limite inferior & Peso sugerido & Limite superior \\
\hline Interno-Externo & 50,02 & 62,50 & 66,64 \\
\hline Externo-Interno & 33,37 & 37,50 & 49,98 \\
\hline
\end{tabular}

Comutação $=62,50 * \mathrm{IE}+37,50 * \mathrm{EI}$

\subsection{Avaliação dos processos}

A etapa de avaliação dos processos é conduzida de maneira análoga às etapas anteriores. Os julgamentos de diferença de atratividade são emitidos (par a par) para os seis processos identificados na Figura 1, quais sejam, Manutenção do acervo (MANUT); Processamento Técnico (PROCTEC); Referência (REFER); Circulação (CIRC); Comutação Marketing/Divulgação (MKTDV).

Nessa etapa foi utilizado um procedimento adicional para auxiliar o 
decisor a estabelecer a ordem decrescente de atratividade dos processos. Nesta etapa de ordenação a pergunta realizada é, por exemplo a seguinte: Estando os processos de Manutenção do acervo $e$ Processamento técnico ambos no nível neutro, seria mais atrativo passar para o nível bom no processo de Manutenção do acervo ou no de Processamento técnico, mantendo-se os outros processos no nível neutro?

Cada elemento da matriz é preenchido com 0 ou 1, dependendo da resposta à pergunta: 1 para o caso de ser atrativo a mudança de nível do processo $X$ e 0 caso contrário. A ordenação dos processos é feita somando-se o valor dos elementos de cada linha; quanto maior o somatório da linha, mais atrativo é o processo. A Figura 9 mostra os resultados para esta etapa.

Assim, a ordem de atratividade atribuída pelo decisor aos seis processos é: Referência (REFER); Comutação (COMUT); Circulação (CIRC); Manutenção do acervo (MANUT); Processamento Técnico (PROCTEC); Marketing/Divulgação (MKTDV).

A Tabela 8 traz os pesos resultantes e a expressão (9) mostra a relação matemática entre os processos realizados pela biblioteca.

\begin{tabular}{|l|c|c|c|c|c|c|c|}
\cline { 2 - 8 } \multicolumn{1}{c|}{} & MANUT & PROCTEC & REFER & CIRC & COMUT & MKTDV & Soma \\
\hline MANUT & 0 & 1 & 0 & 0 & 0 & 1 & 2 \\
\hline PROCTEC & 0 & 0 & 0 & 0 & 0 & 1 & 1 \\
\hline REFER & 1 & 1 & 0 & 1 & 1 & 1 & 5 \\
\hline CIRC & 1 & 1 & 0 & 0 & 0 & 1 & 3 \\
\hline COMUT & 1 & 1 & 0 & 1 & 0 & 1 & 4 \\
\hline MKTDV & 0 & 0 & 0 & 0 & 0 & 0 & 0 \\
\hline
\end{tabular}

Figura 9: Ordenação dos processos por atratividade.

Tabela 8: Pesos para os serviços realizados pela biblioteca.

\begin{tabular}{|l|c|c|c|}
\cline { 2 - 4 } \multicolumn{1}{c|}{} & Limite inferior & Peso sugerido & Limite superior \\
\hline Referência & 22,02 & 22,39 & 22,76 \\
\hline Comutação & 18,71 & 19,90 & 20,28 \\
\hline Circulação & 18,22 & 18,41 & 19,59 \\
\hline Manutenção do acervo & 16,02 & 16,42 & 16,81 \\
\hline Processamento Técnico & 14,23 & 14,43 & 14,83 \\
\hline Marketing/Divulgação & 8,02 & 8,46 & 8,89 \\
\hline
\end{tabular}

Serviços da biblioteca $=22,39 *$ REFER $+19,90 *$ COMUT $+18,412 *$ CIRC +

$$
16,42 * \text { MANUT }+14,43 * \text { PROCTEC }+8,46 * \text { MKTDV }
$$

\subsection{Discussão}

A visualização em forma de árvore dos serviços realizados pela biblioteca permite uma percepção mais acurada de todos os processos e subprocessos e de seus relacionamentos.

A etapa de ordenação dos processos permite, a priori, estabelecer uma hierarquização das atividades. Esta etapa pode ser importante nos direcionamentos de prioridades a serem executadas pela biblioteca (o processo ou subprocesso de maior atratividade deve receber atenção prioritária).

ENGEVISTA, v. 7, n. 1, p. 21-31, abril 2005
A etapa de obtenção dos pesos tem duas finalidades básicas. A primeira é a obtenção de um índice ponderado de produção, que pode expressar com maior fidelidade a produção (ou produtividade, se for o caso) de cada biblioteca.

Caso houvesse informações quantificadas sobre cada um dos serviços prestados pelas bibliotecas e sobre os recursos usados, modelos tipo DEA (COOPER et al., 2000) poderiam ser empregados para medir a eficiência relativa das bibliotecas da Embrapa. Nesse caso, o índice de produção 
proposto neste artigo poderia ser usado como o output do modelo DEA.

\section{CONCLUSÕES}

Este artigo traz uma primeira aproximação para o estudo da importância relativa de serviços de bibliotecas, com o uso de modelos de Pesquisa Operacional, a saber, modelos do Apoio Multicritério à Decisão, com o objetivo de gerar um índice de produção de serviços de bibliotecas. Pode-se julgar inadequado interagir com somente um único decisor emitindo julgamentos de valor e que os resultados seriam melhor sustentados se baseados em opiniões de múltiplos decisores ou por um processo de questionários. Entretanto, destaca-se que optou-se pela opinião de um único decisor que refletiu intensamente e que conhecia bastante o assunto, ao invés de usar a opinião de diversos peritos que poderiam emitir julgamentos superficiais.

Essa metodologia pode ser bastante útil e adequada para a avaliação de serviços de bibliotecas. Tanto a etapa de estruturação do problema, com a eleição dos serviços e de subserviços, quanto a emissão de juízos de valor dependem da interação com o decisor. Decisores diferentes podem gerar famílias de critérios e importâncias relativas diferentes.

Os pesos, além de serem usados quantitativamente na composição do índice de produção de serviços, podem ser usados como ferramentas de apoio à gestão, já que pesos mais elevados significam maior importância relativa do serviço, que deve ser priorizado quando há conflitos na gestão dos serviços.

Finalmente, o índice proposto pode servir como medida de comparação de produção de serviços entre diversas bibliotecas, em especial quando estão sujeitas a uma administração central, que necessita de resultados objetivos para apoiar suas decisões e como ferramenta de planejamento estratégico, em especial se usado como variável de um modelo DEA. Entretanto, ressalta-se que para seu uso efetivo, os julgamentos de valor de outros bibliotecários (posteriormente combinados) devem ser considerados.

\section{REFERÊNCIAS}

BANA E COSTA C.A.; CHAGAS, M.P. A career choice problem: an example of how to use macbeth to build a quantitative value model based on qualitative value judgments. European Journal of Operational Research, v. 153, n. 2, p. 323-331, 2004.

BANA E COSTA, C.A., DE CORTE, J.M., VANSNICK, J.C. MACBETH. Working Paper LSEOR 03.56. London: London School of Economics, 2003.

BANA E COSTA, C.A., DE CORTE, J.M., VANSNICK, J.C. On the Mathematical Foundations of Macbeth. Working Paper LSEOR 03.61. London: London School of Economics, 2004.

BANA E COSTA, C.A., FERREIRA, J.A.A, CORREA, E.C. Metodologia multicritério de apoio à avaliação de propostas em concursos públicos. In: ANTUNES, C.H., TAVARES, L.V. (Eds.) Casos de Aplicação da Investigação Operacional, Amadora: McGraw Hill, 2000, p. 336-363.

BANA E COSTA, C.A., VANSNICK, J.C. A fundamental criticism to Saaty's use of the eigenvalue procedure to derive priorities. Working Paper LSEOR 01.42. London: London School of Economics, 2001.

BANA E COSTA, C.A., VANSNICK, J.C. Thoughts on a theoretical framework for measuring attractiveness by categorical based evaluation technique (MACBETH). In: CLÍMACO, J. (Ed.) Multicriteria Analysis, Berlin: SpringerVerlag, 1997.

BANA E COSTA, C.A., VANSNICK, J.C. Uma nova abordagem ao problema da construção de uma função de valor cardinal: MACBETH. Investigação Operacional, v. 15, p. 15-35, 1995. BUCKLAND, M. Library services in theory and context. 2.ed. Michael Buckland, 1999.

BUCKLAND, M. Redesigning library services: a manifesto. American Library Association, 1992. 
COOPER, W.W.; SEIFORD, L.M.; TONE, K. Data Envelopment Analysis:

A Comprehensive Text with Models, Applications, References and DEASolver Software. New York: Kluwer Academic Publishers, 2000.

DIAS, L.M.C., ALMEIDA, L.M.A.T., CLÍMACO, J. Apoio Multicritério à

Decisão, Coimbra: Universidade de Coimbra, 1996.

GOMES, E.G. Integração entre Sistemas de Informação Geográfica e Métodos Multicritério no Apoio à Decisão Espacial. 1999. Tese (Mestrado em Engenharia de Produção) - COPPE, Universidade Federal do Rio de Janeiro, Rio de Janeiro.

GOMES, L.F.A.M, GOMES, C.F.S., ALMEIDA, A.T. Tomada de decisão gerencial: enfoque multicritério. São Paulo: Editora Atlas, 2002.

GOMES, L.F.A.M., GONZALEZARAYA, M.C., CARIGNANO, C. Tomada de decisões em cenários complexos. São Paulo: Pioneira Thompson Learning, 2004.

MUELLER, S.P.M.; PECEGUEIRO, C.M.P.de A. O periódico Ciência da Informação na década de 90: um retrato da área refletido em seus artigos. Ciência da Informação, Brasília, v. 30, n. 2, p. 47-63, 2001.

POMEROL, J.C., BARBA-ROMERO, S. Multicriterion Decision in Management: Principles and Practice. Boston: Kluwer Academic Publishers, 2000.

ROY, B., BOUYSSOU, D. Aide multicritère à la décision: méthods et cas. Paris: Economica, 1993.

SAATY, T.L. The Analytic Hierarchy Process. New York: McGraw Hill Company, 1980.

SOARES DE MELLO, J.C.C.B.S, GOMES, E.G., LETA, F. R., PESSOLANI, R.B.V. Conceitos básicos do Apoio Multicritério à Decisão e sua aplicação no Projeto Aerodesign. Engevista, v. 5, n. 8, p. 22-35, 2003.

YU, P.L. Multiple criteria decision making: concepts, techniques and extensions. New York: Plenum Press, 1985. 